\title{
Trend of Sensitivity Pattern of Uropathogenic Escherichia coli: Five Year Experience at a Tertiary Care Hospital in Dhaka
}

\author{
Begum $\mathrm{F}^{1}$, Mosaddek $\mathrm{ASM}^{2}$, Perveen $\mathrm{K}^{3}$, Karim $\mathrm{R}^{4}$, Begum $\mathrm{NN}^{5}$
}

\begin{abstract}
Background: Urinary tract infections are among the most common bacterial infections caused by pathogens with a decreasing susceptibility to several classes of antimicrobials. Objective: The purpose of the present study was to see the trend of sensitivity pattern of Escherichia coli (E. coli) isolated from the urinary tract patients. Methodology: This retrospective study was conducted at Uttara Adhunik Medical College Hospital, Dhaka from 2008 to 2012 for a period of 5(five) years. All patients were within ages 1 month to 80 years, comprising of both male and female, either out or in patients were included as study population. Urine sample was collected and Escherichia coli was isolated and identified as well as the antimicrobial susceptibility patterns was determined. Results: A total number of 16,666 urine samples were collected. No significant changes of susceptibility pattern of Escherichia coli was found to ciprofloxacin, cephradine, ceftriaxone, levofloxacin, nalidixic acid, imipenem and meropenem; however, significant change was detected to amoxicillin, amoxiclav, cefixime, trimethoprim-sulfamethoxazole, amikacin, ceftazidime and mecillinam between 2008 and 2012. Conclusion: Trend of sensitivity pattern of Escherichia coli is changing to few important antibiotics. [J Shaheed Suhrawardy Med Coll, 2013;5(2):103-105]
\end{abstract}

Keywords: Urinary tract infection, uropathogens, Escherichia coli, antimicrobial susceptibility

Received: March 2013; Revised: April 2013; Accepted: May 2013

\section{Introduction}

Increasing rates of resistance among bacterial uropathogens has become a major public health problem in both developed and developing countries ${ }^{1}$. Several factors are associated with the rise of resistance rate of bacterial uropathogens including misuse of antimicrobials ${ }^{2}$, frequent oral use of wide-spectrum antimicrobials that may change the intestinal flora, which is usually common cause of urinary tract infection ${ }^{2-4}$ and inappropriate dosages and duration of treatment ${ }^{5}$. Escherichia coli is the primary urinary tract pathogen, accounting for 75 to $90 \%$ of uncomplicated urinary tract infection isolates ${ }^{4}$. Surveillance studies provide information of the causative agents of UTIs and their antimicrobial resistance patterns which may aid clinicians in choosing the appropriate antimicrobial empirical treatment.

This study was conducted to determine the antimicrobial susceptibility patterns of Escherichia coli towards ciprofloxacin, trimethoprim-sulfamethoxazole, ceftriaxone, cephradine, ceftazidime, levofloxacin, imipenem, meropenem, amikacin, cefuroxime, amoxicillin, amoxiclav, cefixime, and mecillinam during a 5-year period from January 2008 to December 2012.

\section{Methodology}

This retrospective study was carried out at Uttara Adhunik Medical College Hospital, Dhaka, Bangladesh from January 2008 to December 2012 on patients who presented symptoms

1. Prof. Dr. Most. Fahmida Begum, Professor \& Head, Department of Microbiology, Uttara Adhunik Medical College, Dhaka

2. Prof. Dr. Abu Syed Md. Mosaddek, Professor \& Head, Department of Pharmacology, Uttara Adhunik Medical College, Dhaka

3. Prof. Dr. Kawser Perveen, Professor \& Head, Department of Pathology, Uttara Adhunik Medical College, Dhaka

4. Dr. Rezina Karim, Associate Professor, Department of Microbiology, Uttara Adhunik Medical College, Dhaka

5. Dr. Nurun Nahar Begum, Associate Professor, Department of Microbiology, Uttara Adhunik Medical College, Dhaka

\section{Correspondence}

Prof. Dr. Most. Fahmida Begum, Professor \& Head, Department of Microbiology, Uttara Adhunik Medical College, Uttara, Dhaka, Bangladesh; Email: fahmidabelly1994@yahoo.com; Cell No: +8801615457899 
of urinary tract infections. All patients were within ages 1 month to 80 years, comprising of both male and female, either outpatient or inpatients department were included in this study. The diagnosis of urinary tract infection was based on microscopic findings of more than 5 White blood cells per high power field (1000x for high power) and a colony count of $105 \mathrm{CFU} / \mathrm{ml}$ of a single pathogen. The urine of all patients was sampled by clean catch of midstream urine. Urine samples were delivered to the laboratory within 1 hour of collection and processed within 2-4 hours.

Isolation and identification of Escherichia coli: A loopful $0.01 \mathrm{ml}$ of urine sample was cultured on blood agar (Oxoid N.Y., USA) and MacConkeys agar (Oxoid N.Y., USA) and was incubated for 24 hours at 37?C aerobically. Escherichia coli was selected for inclusion in further study when it was isolated as pure culture as well as the concentrations was greater than $105 \mathrm{CFU} / \mathrm{ml}$. Isolations and identifications were performed by biochemical tests.

Antimicrobial susceptibility testing: Kirby Bauer disc diffusion technique ${ }^{6}$ was used and 0.5 MacFarland's 108/mL employed in inoculums suspensions preparation according to the recommendations of the Clinical Laboratory Standard Institute (CLSI), former the National Committee for Clinical Laboratory Standards (NCCLS) $)^{7-8}$. The discs were ciprofloxacin, trimethoprim-sulfamethoxazole, ceftriaxone, cephradine, ceftazidime, levofloxacin, imipenem, meropenem, amikacin, cefuroxime, amoxicillin, amoxiclav, and cefixime (Table I) and were tested against the isolates. Sensitivity test was performed by disc diffusion technique using commercially available discs on Mueller Hinton agar (Oxoid N.Y., USA) plates?.

Statistical analysis: Statistical analysis was done by $\mathrm{Z}$ test.

Table I: Antibiotics Disc (Oxoid N.Y., USA) Used for the Disc diffusion technique against Escherichia coli

\begin{tabular}{lccc}
\hline Antibiotic & Conc. level & Antibiotic & Conc. level \\
\hline $\begin{array}{l}\text { Trimethoprim- } \\
\text { sulfamethoxazole }\end{array}$ & $25 \mathrm{~g}$ & Mecillinam & $25 \mathrm{~g}$ \\
Cefuroxime & $30 \mathrm{~g}$ & Ceftazidime & $30 \mathrm{~g}$ \\
Amoxiclav & $30 \mathrm{~g}$ & Amikacin & $30 \mathrm{~g}$ \\
Cephradine & $30 \mathrm{~g}$ & Ceftriaxone & $30 \mathrm{~g}$ \\
Amoxicillin & $1010 \mathrm{~g}$ & Cefixime & $05 \mathrm{~g}$ \\
Levofloxacin & $05 \mathrm{~g}$ & Ciprofloxacin & $05 \mathrm{~g}$ \\
Meropenem & $10 \mathrm{~g}$ & Imipenem & $10 \mathrm{~g}$ \\
\hline
\end{tabular}

*Commercial antibiotics discs (Oxoid N.Y., USA) were used.

\section{Results}

A total number of 16, 666 reports of urine samples were collected from the microbiology laboratory data base of which 3,000(18\%) reports showed presence of E. coli. E. coli were mostly susceptible to Meropenem from the year 2008 to $2012(100 \%)$ except $2010(98.58 \%)$ followed by amikacin (81.20\%-100\%) and imipenem (78.66\%-100\%). Gradual decrease of susceptibility pattern of mecillinam was found from 2008-9 (3.27\%) to $2012(75.65 \%)$ except 2010 where slight increases of sensitivity occur than 2009. Amoxicillin is highly resistant $(0.0-7.7 \%)$ to $E$. coli. Resistant pattern was gradually increased in case of cephradine and amoxiclav which was $32.48 \%$ in $2008,0.57 \%$ in 2012 and $80.28 \%$ in $2008,35.56 \%$ in 2012 . Study showed no significant changes of susceptibility pattern of this pathogen to ciprofloxacin, cephradine, ceftriaxone, levofloxacin, cefuroxime and nalidixic acid; however, significant change of $p$ value ( $p$ $<0.05$ ) to amoxicillin, amoxiclav, cefixime, amikacin, ceftazidime and mecillinam between 2008 and 2012. Increase susceptibility of $E$. coli to trimethoprim-sulfamethoxazole occur from $2008(41.76 \%)$ to $2012(58.89 \%)(p<0.05)$.

Table 2: Antimicrobial Sensitivity patterns of E. coli in urine isolated from the years 2008 to 2012

\begin{tabular}{lccccc}
\hline Antibiotics & $\mathbf{2 0 0 8}$ & $\mathbf{2 0 0 9}$ & $\mathbf{2 0 1 0}$ & $\mathbf{2 0 1 1}$ & $\mathbf{2 0 1 2}$ \\
& $\mathbf{n = 4 3 1}$ & $\mathbf{n = 4 7 2}$ & $\mathbf{n = 5 6 4}$ & $\mathbf{n = 6 7 0}$ & $\mathbf{n = 8 8 3}$ \\
\hline Ciprofloxacin & $192(44.55)$ & $258(54.66)$ & $288(51.06)$ & $305(45.52)$ & $435(49.26)$ \\
Cephradine & $140(32.48)$ & $162(34.32)$ & $96(17.02)$ & $43(06.42)$ & $05(00.57)$ \\
Ceftriaxone & $236(54.76)$ & $275(58.26)$ & $344(60.99)$ & $368(54.92)$ & $484(54.81)$ \\
Levofloxacin & $257(59.63)$ & $263(55.72)$ & $288(51.06)$ & $326(48.66)$ & $442(50.06)$ \\
TMX & $180(41.76)$ & $231(48.94)$ & $293(51.95)$ & $279(41.64)$ & $520(58.89)$ \\
Imipenem & $419(97.21)$ & $472(100)$ & $559(99.11)$ & $527(78.66)$ & $881(99.77)$ \\
Meropenem & $431(100)$ & $472(100)$ & $556(98.58)$ & $670(100)$ & $883(100)$ \\
Amikacin & $379(87.93)$ & $454(96.19)$ & $458(81.20)$ & $590(88.06)$ & $883(100)$ \\
Ceftazidime & $289(67.05)$ & $379(80.30)$ & $415(73.58)$ & $449(67.01)$ & $503(56.96)$ \\
Amoxicillin & $33(07.66)$ & $25(05.30)$ & $24(04.25)$ & $20(02.98)$ & $00(00)$ \\
Nalidixic acid & $87(20.18)$ & $95(20.13)$ & $192(34.04)$ & $132(19.70)$ & $107(12.12)$ \\
Amoxiclav & $346(80.28)$ & $324(68.64)$ & $338(59.93)$ & $361(53.88)$ & $314(35.56)$ \\
Cefixime & $216(50.12)$ & $261(55.30)$ & $324(57.45)$ & $316(47.16)$ & $330(37.37)$ \\
Cefuroxime & $217(50.35)$ & $215(45.55)$ & $324(57.45)$ & $242(36.12)$ & $444(50.28)$ \\
Mecillinam & $402(93.27)$ & $425(90.04)$ & $514(91.13)$ & $563(84.03)$ & $668(75.65)$ \\
\hline
\end{tabular}

* Figure within the parenthesis indicates percentage.

* $\mathrm{TMX}=$ trimethoprim - Sulfamethoxazole

\section{Discussion}

More than $95 \%$ of UTIs are caused by a single bacterial species ${ }^{10}$. Pathogens causing UTIs are almost always predictable, with $E$. coli the primary etiologic agent among both outpatients and inpatients. Infectious Diseases Society of America (IDSA) ${ }^{11}$ recently recommended that each hospital should establish routine mechanisms to determine the local resistance rates among uropathogens and that the standard antimicrobial regimens for empirical treatment of UTIs should be reassessed periodically in light of changing susceptibility patterns. Physicians should be aware of current antimicrobial susceptibility patterns for $E$. coli and other uropathogens in their local communities as antimicrobial susceptibility changes over time ${ }^{12}$.

As regards antimicrobial susceptibility pattern, E. coli are poorly susceptible to Amoxicillin $(07.56 \%$ - $0 \%)$ and Cephradine $(32.48 \%$ to $00.57 \%)$ Trimethoprimsulfamethoxazole (41.76-58.89\%) throughout this period (2008-2012). Similar observation was reported in other authors from Pakistan ${ }^{13,14,15,16}$. Susceptibility pattern of Trimethoprim-sulfamethoxazole is increasing due to less use of this drug in clinical practice in UTI.

Fluoroquinolones are a logical choice for the empirical treatment of uncomplicated UTIs; however, their 
widespread use has raised concern for increasing fluoroquinolone resistance ${ }^{17}$ which is similar in case of Levofloxacin but in Ciprofloxacin susceptibility rate was increasing over the years. Pattern of susceptibility to Ceftriaxone were almost homogenous in this study over last five years. The activity Nalidixic acid against $E$. coli has decreased significantly over six years $(2006-2011)^{18}$ similar to this study.

E. coli showed highest sensitivity towards imipenem $(97.21 \%$ to $99.77 \%)$, meropenem $(100 \%)$ and amikacin $(87.93-100 \%)$ which correlates with the study of Tanvir et $\mathrm{al}^{19}$.

Gradual improvement in susceptibility of $E$. coli was to Cefixime during a study period of 2005 to 2009 differs from this study where resistant pattern is increased but similar to study on Amoxicillin-clavulanic acid where gradual decline of susceptibility ${ }^{20}$.

\section{Conclusion}

Higher resistance rates to all antibiotics tested in our study may be explained by high and uncontrolled consumption of these antibiotics during the past decade in our institute. All antimicrobials are available as over-the-counter drugs without requiring the doctor prescriptions in our country. A good infection control and antibiotic policy will certainly help in delaying the era of unabated microorganisms for which no antibiotic is going to be effective.

\section{References}

1. Dromigny JA, Nabet P, Gros-Claude JD. Risk factors for antibioticresistant Escherichia coli isolated from community-acquired urinary tract infections in Dakar, Senegal. J Antimicrob Chemother 2005; 56:236-9.

2. Col NF, O'Connor RW. Estimating worldwide current antibiotic usage: report of task force 1. Rev Infect Dis 1987;9(Suppl.3):232-43

3. Winberg J, Bergstrom T, Lincoln K, Lidin-Janson G. Treatment trials in urinary tract infection (UTI) with special reference to the effect of antimicrobials on the fecal and periurethral flora. Clinical nephrology. 1973;1(3):142-8

4. Edlund C, Nord C. Effect on the human normal microflora of oral antibiotics for treatment of urinary tract infections. J Antimicrob Chemother 2000; 46:41-8.

5. Bhutta ZA. Drug resistant infections in poor countries: A major burden on children. BMJ 2008; 336(7650):948-9.
6. Bauer AW, Kirby WM, Sherris JC, Jurck M. Antibiotic susceptibility testing by a standard single disc method. American Journal Clinical Pathology.1996; 451:493-496

7. National Committee for Clinical Laboratory Standards 1999. Performance standards for antimicrobial susceptibility testing, Ninth informational suppl Vol 18:1 National committee for clinical laboratory standards, Wyne Pa.

8. Murray Patrick Elln Baron, James Jorgensen, Michael A Pfaller, Robert H Yorken, 2003. Susceptibility testing methods yeast and filamentous fungi, manual of clinical microbiology 8th ed. Vol 2 American Society Microbiology press Washington DC.

9. Iroha IR, Adikwu MU, Esimone CO, Aibinu I, Amadi ES. Extended spectrum Beta- Lactamase (EBSL) in E. coli isolated from a tertiary hospital in Enugu state, Nigeria Pak. Journal of Medical Sciences 2009; 25(2): 279-282.

10. Winstanley TG, Limb DI, Eggington R, Hancock F. A 10-year survey of the antimicrobial susceptibility of urinary tract isolates in the UK: the Microbe Base project. J Antimicrob Chemother 1997; 40:591-4.

11. Nicolle LE. Epidemiology of urinary tract infection. Infect Med 2001; 18:153-62.

12. Sahm DF, Thornsberry C, Mayfield DC, Jones ME, Karlowsky JA. Multidrug-resistant urinary tract isolates of Escherichia coli: prevalence and patient demographics in the United States in 2000. Antimicrob Agents Chemother 2001; 45:1402-6.

13. Ahmad SI, Zafar T, Farooqui S, et al, Urine examination of 1460 patients suspected of urine tract infection. J Pak Med Assoc 1975; 25:169-70

14. Farooqi BJ, Khursheed M, Alam M. Urinary tract infection. J Pak Med Assoc 1989; 25:129-31.

15. Nizami SQ, Khan IA, Farooqui B. Treatment of UTI in children in Karachi, Pakistan: which antibiotic to use? Infect Dis J Pak 1997:25-6.

16. Abbas JA, Ashiq B, Burney MI. Bacteriuria in child. J Pak Med Assoc $1975 ; 25: 169$

17. Warren JW, Abrutyn E, Hebel JR, Johnson JR, Schaeffer AJ, Stamm WE. Guidelines for antimicrobial treatment of uncomplicated acute bacterial cystitis and acute pyelonephritis in women. Infectious Diseases Society of America (IDSA). Clin Infect Dis 1999; 29:745-58.

18. Schaeffer AJ, Cao N, Rajan Q, Anderson EB, Pruden LD, Sensibar J, Duncan LJ. Host pathogenesis in urinary tract infection. Int $\mathrm{J}$ Antimicrob Agts 2001;17: 245-251

19. Tanvir R, Hafeez R, Hasnain S. Prevalence of Multiple Drug Resistant Escherichia coli in Patients of Urinary tract infection Registering at a Diagnostic Laboratory in Lahore Pakistan. Pak J Zool 2012;44:707-12.

20. Iqbal J, Rahman M, Kabir MS, Rahman M. Increasing ciprofloxacin resistance among prevalent urinary tract bacterial isolates in Bangladesh. Jpn J Med Sci Biol 1997;50(6): 241-250 